\title{
The Atmospheric Monitoring System of the JEM-EUSO space mission
}

\author{
M.D. Rodríguez Frías ${ }^{1,2}$, S. Toscano ${ }^{2}$, E. Bozzo $^{2}$, L. del Peral ${ }^{1,2, a}$, A. Neronov ${ }^{2}$, \\ and $\mathrm{S}$. Wada ${ }^{3}$ for the JEM-EUSO Collaboration \\ ${ }^{1}$ SPace \& AStroparticle (SPAS) Group, UAH, Madrid, Spain \\ ${ }^{2}$ ISDC, Astronomy Dept. University of Geneva, Versoix, Switzerland \\ ${ }^{3}$ RIKEN Advanced Science Institute, Japan
}

\begin{abstract}
An Atmospheric Monitoring System (AMS) is a mandatory and key device of a space-based mission which aims to detect Ultra-High Energy Cosmic Rays (UHECR) and Extremely-High Energy Cosmic Rays (EHECR) from Space. JEM-EUSO has a dedicated atmospheric monitoring system that plays a fundamental role in our understanding of the atmospheric conditions in the Field of View (FoV) of the telescope. Our AMS consists of a very challenging space infrared camera and a LIDAR device, that are being fully designed with space qualification to fulfil the scientific requirements of this space mission. The AMS will provide information of the cloud cover in the FoV of JEM-EUSO, as well as measurements of the cloud top altitudes with an accuracy of $500 \mathrm{~m}$ and the optical depth profile of the atmosphere transmittance in the direction of each air shower with an accuracy of 0.15 degree and a resolution of $500 \mathrm{~m}$. This will ensure that the energy of the primary UHECR and the depth of maximum development of the EAS ( Extensive Air Shower) are measured with an accuracy better than 30\% primary energy and $120 \mathrm{~g} / \mathrm{cm}^{2}$ depth of maximum development for EAS occurring either in clear sky or with the EAS depth of maximum development above optically thick cloud layers. Moreover a very novel radiometric retrieval technique considering the LIDAR shots as calibration points, that seems to be the most promising retrieval algorithm is under development to infer the Cloud Top Height (CTH) of all kind of clouds, thick and thin clouds in the FoV of the JEM-EUSO space telescope.
\end{abstract}

\section{Introduction}

Cosmic Ray Physics is one of the fundamental key issues and an essential pillar of Astroparticle Physics that aims, in a unique way, to address many fundamental questions of the non-thermal Universe in the Astroparticle Physics domain. The huge physics potential of this field can be achieved by an upgrade of the performances of current ground-based experiments and pioneer spacebased missions, as the JEM-EUSO space telescope [1]. The JEM-EUSO space mission is the Extreme-Universe Space Observatory (EUSO) which will be located at the Exposure Facility of the Japanese Experiment Module $(\mathrm{JEM} / \mathrm{EF})$ on the International Space Station (ISS) and looking downward the atmosphere will allow a full-sky monitoring capability to watch for Ultra-High Energy Cosmic Rays (UHECR) and Extremely High Energy Cosmic Rays (EHECR). An Atmospheric Monitoring System (AMS) is mandatory and a key element of a Spacebased mission which aims to detect Ultra-High Energy Cosmic Rays (UHECR). JEM-EUSO has a dedicated atmospheric monitoring system that plays a fundamental role in our understanding of the atmospheric conditions in the FoV of the main telescope. The JEM-EUSO AMS consists of a bi-spectral infrared camera and a LIDAR device that are being fully designed under space qualification to fulfil the scientific requirements of this space mission.

\footnotetext{
a e-mail: luis.delperal@gmail.com
}

\section{The Atmospheric Monitoring System}

To fully monitor the atmosphere and to retrieve the cloud coverage and cloud top height in the JEM-EUSO FoV, an Atmospheric Monitoring System (AMS) is foreseen in the telescope. The AMS [2] is crucial to estimate the effective UHECR \& EHECR exposure of the telescope and for the proper analysis of the UHECR \& EHECR events under cloudy conditions $[3,4]$.

The AMS of JEM-EUSO will include:

1. a bi-spectral Infrared (IR) camera;

2. a LIght Detection And Ranging (LIDAR) device;

3. global atmospheric models generated from the analysis of all available meteorological data by global weather services such as the National Centers for Environmental Predictions (NCEP), the Global Modeling and Assimilation Office (GMAO) and the European Centre for MediumRange Weather Forecasts (ECMWF) [5];

4. the slow mode data of JEM-EUSO, the monitoring of the pixel signal rate every $3.5 \mathrm{~s}$ for the observation of Transient Luminous Events (TLEs), which will give additional information on cloud distribution and the intensity of the night sky airglow.

The JEM-EUSO telescope will observe the EAS development only during night time. The IR camera will cover the entire FoV of the telescope in order to detect the presence of clouds and to obtain the cloud cover and the cloud top altitude. The LIDAR will be shot in some pre-defined

This is an Open Access article distributed under the terms of the Creative Commons Attribution License 4.0, which permits unrestricted use, distribution, and reproduction in any medium, provided the original work is properly cited. 


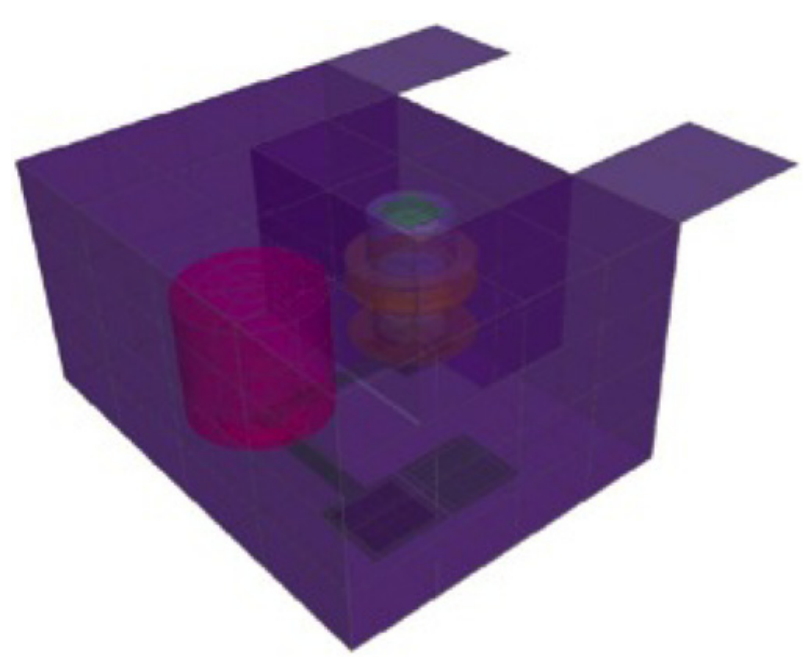

Figure 1. IR Camera Preliminary Design Modelization.

position around the location of triggered EAS events. The LIDAR will be used to measure the clouds altitude and optical depth as well as the optical depth vertical profile of the atmosphere along these directions with a range accuracy of $375 \mathrm{~m}$ in nadir. The IR camera and the LIDAR have been designed to work in a complementary way.

\section{The Infrared Camera}

The IR Camera (Fig. 1) onboard JEM-EUSO will consist of a refractive optics made of germanium and an uncooled $\mu$ bolometer array detector. The FoV of the IR Camera is $48^{\circ}$, totally matching the FoV of the main JEM-EUSO telescope. The angular resolution, which corresponds to one pixel, is about $0.1^{\circ}$. A temperature-controlled shutter in the camera and blackbodies are used to calibrate background noise and gains of the detector to achieve an absolute temperature accuracy of $\sim 3 \mathrm{~K}$. Therefore, the IR Camera takes images continuously every $17 \mathrm{~s}$ while the ISS moves $1 / 4$ of the FoV of the JEM-EUSO telescope.

A brightness temperature measurement, intended for a single band infrared camera configuration, may not provide the required radiometric accuracy without the use of external information for atmospheric effects correction. Therefore a multispectral approach has been selected as baseline of the Infrared Camera of JEM-EUSO [6]. The delailed and dedicated retrieval algorithm for the cloud top height parameter that fulfill the scientific and technical requierements of the Infrared Camera of JEMEUSO can be found in these Proceedings [4]. In the preliminary design of the IR-Camera a bi-spectral design with two filters in the cold spot of the optics that allows a multispectral snapshot camera without a dedicated filter wheel mechanism is foreseen. This is a very "smart" solution that leads to have a more reliable baseline and to reduce the costs of a complicated filter wheel mechanism intended for Space applications. The only drawback of this solution is that, in order to overcome the use of half of available area of the detector for each spectral band, the IR-Camera images acquisition time has to be faster to avoid gaps during the ISS orbit with the impact on the restricted data budget allocated for an ISS mission.

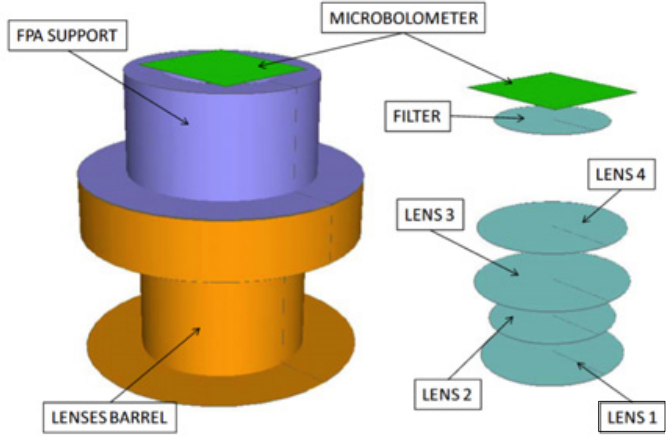

Figure 2. Preliminary Design Scheme of the Optical Unit Assembly.

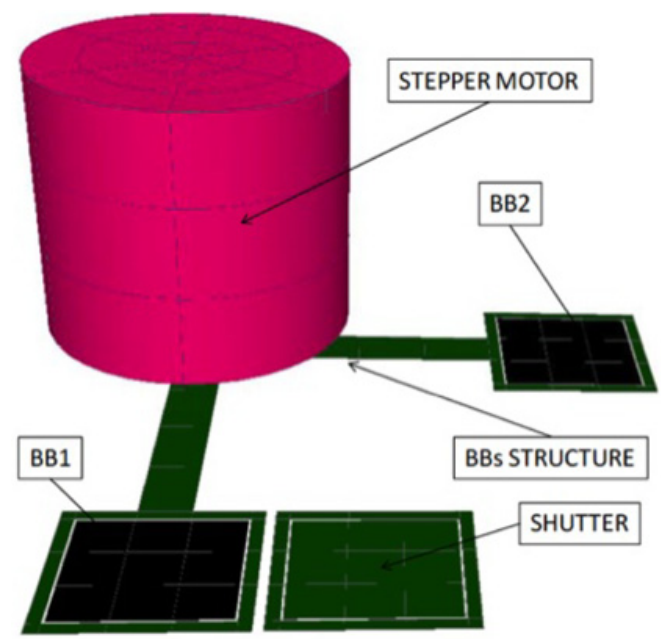

Figure 3. Scheme of the Calibration unit Assembly preliminary design with the two Black Bodies BB1 \& BB2 and the stepper motor.

The preliminary design of the IR Camera [6] can be divided into three main blocks: the Telescope Assembly, the Calibration Unit and the Electronic Assembly. The main function of the Telescope Assembly is to acquire the infrared radiation by means of an uncooled microbolometer and to convert it into digital counts. A dedicated optical design has been developed as well, with a huge angular field to comply with the wide FoV of the JEM-EUSO main telescope (Fig. 2). To assure the high demanding accuracy, a dedicated onboard calibration system is foreseen (Fig. 3). Moreover, this System Preliminary Design is complemented by a challenging Mechanical and Thermal design to secure that the IR-Camera will be completely isolated (Fig. 4).

Meanwhile, the Electronic Assembly provides mechanisms to process and transmit the obtained images, the electrical system, the thermal control and to secure the communication with the platform computer. The Electronics Assembly will be communicated with the Main Instrument (apart from the power input buses) by means of three main links: a Data link, a Command link, and Synchronization lines. In addition, the Electronics Assembly will provide discrete telemetries to the Main Instrument. Regarding the Front End Electronics (FEE), they will be also communicated through serial links, synchronization lines and power generated in the Electronics Assembly to supply the FEE (Fig. 5). 


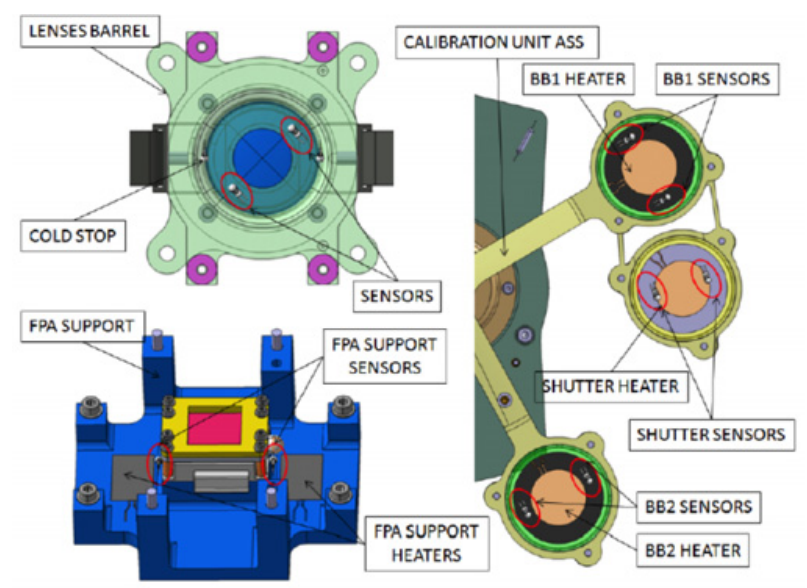

Figure 4. Heaters \& Temperature Sensors Distribution in the System Preliminary Design of the Infrared Camera of JEM-EUSO.
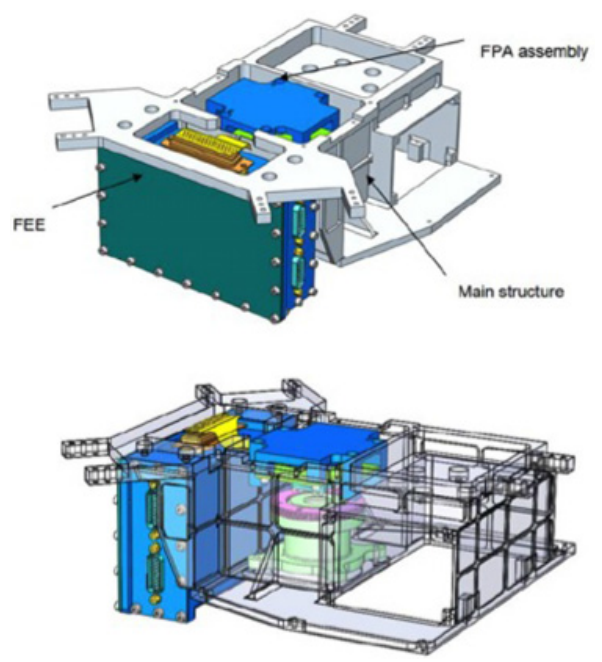

Figure 5. FEE overview of the Infrared Camera.

Moreover, the Interface Control Unit (ICU) can be separated into 2 blocks: the controlling board block and the driving board block. The controlling board block is composed of the electronics board in which will run the communication protocol, using a RS422 type as "telemetry and command" bus. This controlling board is also in charge of the compression algorithm used for the images previously captured by the optics to be sent to the Main Instrument. Assessment of the VHDL code occupation has been made giving as a result that at least a RTAX2000S FPGA from Actel is needed to implement the compression algorithm and the communications protocol. The Driving Board will be composed of the board which contains the driver for the motor and the switches acquisition. Both functionalities will be managed by a small FPGA which runs the control algorithm and drives the actuator. Occupation assessment has been performed, giving as a result that the RTSX72-SU FPGA from Actel is suitable for this design. In addition, a motor will be connected to drivers through relays. These relays will isolate the motor from electronics when stands off. Otherwise, some currents from non-active winding could be driven to the non-active electronics. Driving board will provide power

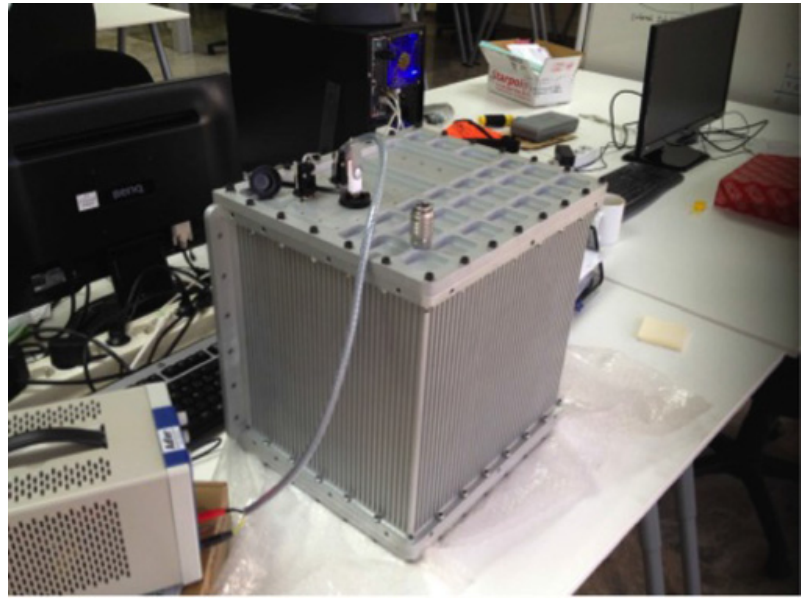

Figure 6. Dedicated bi-spectral and waterproof Infrared Camera for the EUSO-BALLOON (CNES) pathfinder.

to the heaters (32.2 $\Omega$ resistance with a nominal power dissipation of $1.69 \mathrm{~W}$ ) placed in calibration unit by means of a $1 \mathrm{~Hz}$ Pulse Width Modulation (PWM) line of $15 \mathrm{~V}$.

A full characterization and calibration of the Bread Board Model (BBM) for the Infrared Camera of the EUSO-Balloon pathfinder led by CNES [7] was performed on April 2014 at the Instituto de Astrofisica de Canarias (IAC, Tenerife). Moreover this dedicated bi-spectral and waterproof Infrared Camera was flown with EUSOBALLOON first flight on August 24, 2014 from Timmins (Canada) (Fig. 6).

\section{The LIDAR}

The task of the LIDAR is to localize optically thin clouds and aerosol layers and to provide measurements of the scattering and absortion properties of the atmosphere in the region of the EAS development and between the EAS and the JEM-EUSO telescope.

\subsection{LIDAR design}

The LIDAR is composed of a transmission and a receiving system. The transmission system comprises a Nd:YAG laser and a pointing mechanism (PM) to steer the laser beam in the direction of the triggered EAS events. As the laser backscattered signal will be received back by the JEM-EUSO telescope (working as the LIDAR receiver), the laser operational wavelength was chosen to be the third harmonic of the Nd:YAG laser, at $\lambda=355 \mathrm{~nm}$. The laser is being developed at RIKEN (Japan) and will be part of the JAXA (Japanese Space Agency) contribution to the Mission. The PM is under development at the University of Geneva, in close collaboration with $\operatorname{CSEM}^{1}$ (Switzerland).

In the current JEM-EUSO design, the light of the pumping diodes is guided to the laser head through an optical fibre. The PM is conceived to have a steering mirror with two angular degrees of freedom and a maximal tilting angle of $\pm 15^{\circ}$, needed to move the laser beam anywhere within the JEM-EUSO FoV. The LIDAR system will be integrated into the JEM-EUSO telescope. A preliminary

\footnotetext{
${ }^{1}$ www. csem.ch
} 


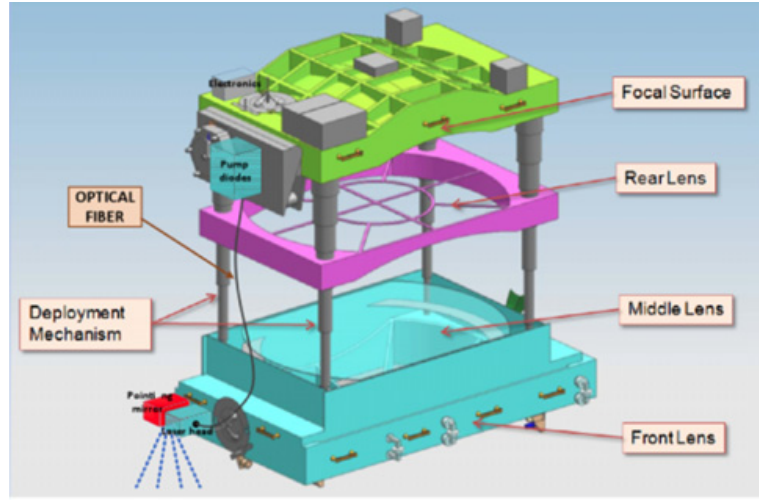

Figure 7. Schematic LIDAR placement on the JEM-EUSO telescope. The laser head and the pointing mirror will be placed close to the front lens of the telescope, while the pump diodes and the control electronics for the mirrors will be placed on the focal surface.

Table 1. Specification for the JEM-EUSO LIDAR.

\begin{tabular}{ll}
\hline Parameter & Specification \\
\hline Wavelength & $355 \mathrm{~nm}$ \\
Repetition Rate & $1 \mathrm{~Hz}$ \\
Pulse width & $15 \mathrm{~ns}$ \\
Pulse energy & $20 \mathrm{~mJ} /$ pulse \\
Beam divergence & $0.2 \mathrm{mrad}$ \\
Receiver & JEM-EUSO telescope \\
Detector & MAPMT (JEM-EUSO) \\
Range resolution (nadir) & $375 \mathrm{~m}$ \\
Steering of output beam & $\pm 30^{\circ}$ from vertical \\
Mass & $14 \mathrm{~kg}$ \\
Dimension & $450 \times 350 \times 250 \mathrm{~mm}$ \\
Power & $<20 \mathrm{~W}$ \\
\hline
\end{tabular}

scheme of the placement of the different elements is shown in Fig. 7. A summary of the specifications needed for the entire system is reported in Table 1.

The LIDAR is expected to receive on average a new trigger on possible EAS events roughly every $\sim 10 \mathrm{~s}$. In the time between two consecutive triggers, the PM should be able to decode the information on the location of the last triggered event within the telescope FoV, re-point the laser beam in this direction, and shoot 5 laser shots covering a sufficiently wide region around the EAS position. The effective time available to the pointing system to steer the laser beam is thus typically of few tenths of seconds, thus requiring a lightweight mirror with limited inertia to optimize the operations of the PM. For this reason, the innovative Micro-Electro-Mechanical Systems (MEMS) technology has been selected by the UniGe to develop the tilting mirror [8]. This mechanism will use magnetic forces to achieve the steering pointing function. A CAD design of the device has been already prepared and is presented in Fig. 8. It consists of a sandwich of two supporting structures that allow a tip-tilt movement of the mirror squeezed in between. The lower supporting structure can be forced in the $\mathrm{X}$ and $\mathrm{Y}$ directions independently by levers connected to it. The ends of these levers are connected to guided magnets that are actuated in the $\mathrm{X}$ and $\mathrm{Y}$

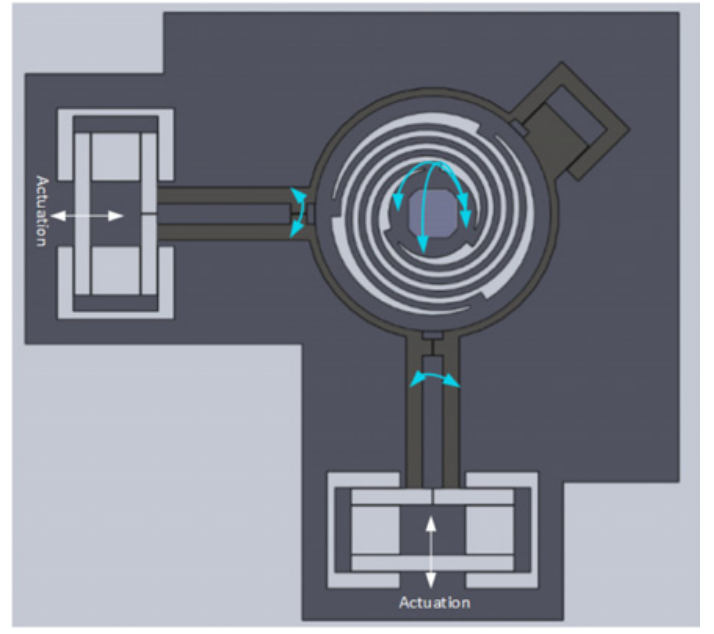

Figure 8. CAD model of the MEMS mirror (not in scale, view from the bottom side). The mirror is squezed between two support structures. It is hold through silicon beamers that allow tilting movements when the levers located on the bottom supporting structure are forced in the $\mathrm{X}$ and $\mathrm{Y}$ direction by the magnetic forces generated by two magnets and the coils controlled by a dedicated electronic board. The coils are not sketched. The two magnets are not visible, but located at the bottom of the actuation points.

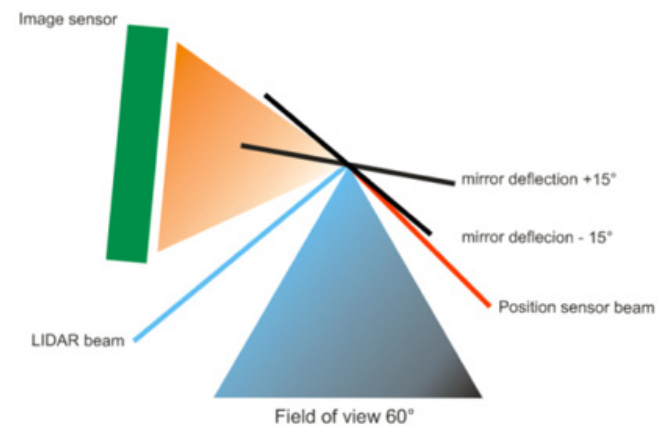

Figure 9. Representation of the laser and position control beam, facing the front side of the MEMS mirror.

directions by coils. The coils will be controlled through an electronic control board, that is also in charge to receive the trigger for possible EAS events and translate this into mirror deplacements. The mirror position is controlled at any time through a high precision positional sensor and the auxiliary optical system. Both the laser and the beam used for the positional sensor will be facing the front side of the mirror, as sketched in Fig. 9. The development of the PM at the UniGe is currently in phase B1. This phase is expected to be completd at the end of 2014 and encompasses the complete design of an Elegant BreadBoard (EBB, see Fig. 10), whose scope is to demonstrate the achievement of the critical PM functionalities. The EBB comprises a commercial version of the laser operating at the same wavelenght of the JEM-EUSO RIKEN laser, an optical system with a position encoder, the MEMS mirror prototype, and the control electronics. The EBB will be implemented during phase B2 and it is expected that a fully operational model will be available at the end of 2015. A qualification and 


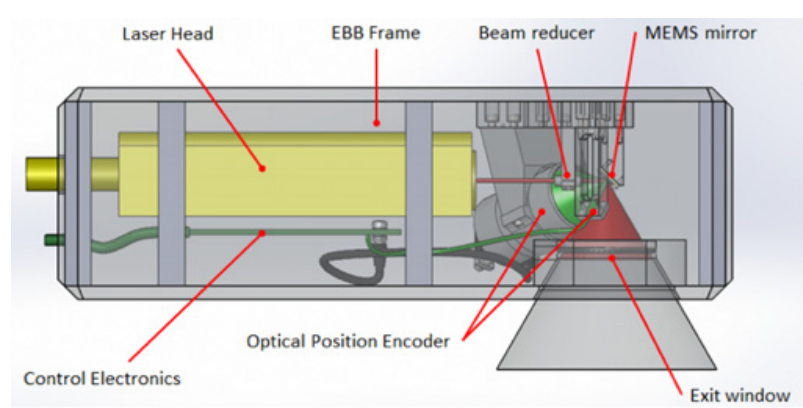

Figure 10. CAD model of the LIDAR PM EBB. In the figure we show the housing, the commercial version of the laser that will be used for the EBB, the optical system supporting the positional sensor, the mems mirror, and the electronic board. All components are represented in scale (the MEMS mirror is about $3 \times 3 \mathrm{~mm}$ large). The red beam represents the laser beam, while the green one is the beam used to measure the position of the MEMS mirror through the positional sensor and the supporting optical system.

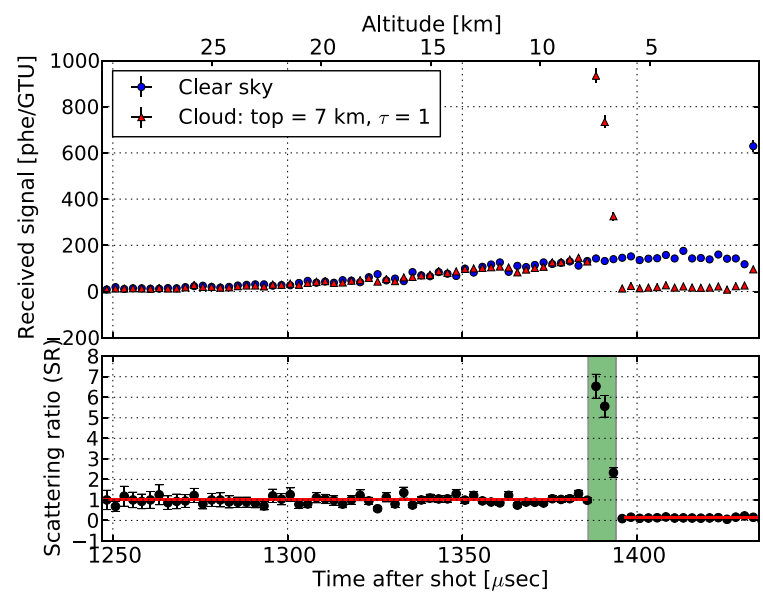

Figure 11. Top: LIDAR backscattered signal in clear sky (blue) and in the presence of an optically thick $(\tau=1)$ cloud (red) as a function of time. Bottom: scattering ratio (SR).

flight model of the JEM-EUSO PM are planned for $>2016$ (project phase C/D).

\subsection{Simulations and data analysis}

Simulations [9] have been carried out in order to study the capability of the system in retrieving the physical properties of atmospheric features such as clouds or aerosol layers. Figure 11 shows an example of the simulated laser backscattered signal detected by the JEMEUSO telescope.

The top panel shows the signal in case of clear atmosphere (blue circles) and in presence of the cloud (red triangles) as a function of the time after shooting the laser and the altitude. The presence of a cloud at $\sim 7 \mathrm{~km}$ will be clearly detected by the LIDAR as an increase of the backscattered signal coming from that region. The bottom panel shows the so-called LIDAR Scattering Ratio (SR), the ratio between the backscattered signal detected in the real condition and a reference profile which represents the backscattered signal in clear atmosphere.

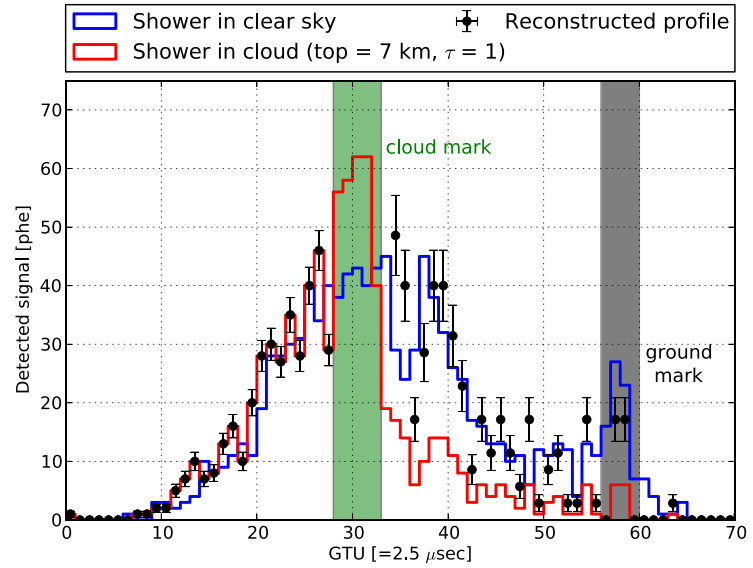

Figure 12. Reconstructed time profile (black points) of $10^{20} \mathrm{eV}$ EAS together with the clear atmosphere (blue) and cloud affected (red) profiles. Error bars are statistical only.

Fitting the SR in the region below the cloud allows for the measurement of the optical depth simply using the formula $\tau=-\log (S R) / 2$.

Once the cloud is detected and its optical depth determined, the cloud-affected EAS profile can be corrected using the formula:

$$
\text { Signal }_{\text {cloud }}=\text { Signal }_{\text {clear }}\left(\exp ^{-\tau}\right)
$$

Figure 12 shows the photons arrival time in $\mathrm{GTU}^{2}$ at the detector focal plane for a shower generated by a UHE proton with $E=10^{20} \mathrm{eV}$ and $\theta=60^{\circ}$. The blue histogram represents the profile of the shower developing in a clear atmosphere, characterised by the presence of the "ground mark" at $\sim 60$ GTU. This feature is due to Cherenkov photons hitting the ground and reflected back to the JEMEUSO focal surface. The red histogram shows the profile of the shower crossing the same optically thick cloud shot by the LIDAR and located at an altitude of $\sim 7 \mathrm{~km}$. As in the case of the LIDAR, the presence of the cloud modifies the EAS time profile, with the appearance of a new feature (the "cloud mark") at 28 GTU, and the ground mark vanishing. After the correction is done using the LIDAR measurement it is possible to retrieve the correct profile (black points) and almost entirely recover the ground mark feature.

\section{Conclusions}

The Infrared Camera and LIDAR of the JEM-EUSO Space Mission are under fully design, prototyping and development under responsability of Japan, Switzerland and Spain. Presently both devices are under Preliminary Design Phase and space qualification of the Infrared Camera and the LIDAR are foreseen to accomplish for the scientific and technical specifications of the JEM-EUSO Space Mission.

\footnotetext{
2 The Gate Time Unit, or GTU, is the time unit of the detector focal surface; $1 \mathrm{GTU}$ corresponds to $2.5 \mu \mathrm{sec}$.
} 
The JEM-EUSO team at the University of Geneva acknowledges support from the Swiss Space Office through a dedicated PRODEX program. This work is supported by the Spanish Government MICINN \& MINECO under the Space Program: projects AYA2009-06037-E/AYA, AYA-ESP 201019082, AYA-ESP 2011-29489-C03, AYA-ESP 2012-39115-C03, AYA-ESP 2013-47816-C4, MINECO/FEDER-UNAH13-4E2741, CSD2009-00064 (Consolider MULTIDARK) and by Comunidad de Madrid under projects S2009/ESP-1496 \& S2013/ICE-2822. M.D. Rodríguez Frías acknowledge International Visitor Grant from the Swiss National Science Foundation (SNSF).

\section{References}

[1] Adams Jr., J.H. et al. (The JEM-EUSO Collaboration), An evaluation of the exposure in nadir observation of the JEM-EUSO mission. Astroparticle Physics, 44, 76, 90 (2013)

Rodríguez Frías, M.D. et al. for the JEM-EUSO Collaboration, The JEM-EUSO Space Mission: Frontier Astroparticle Physics @ ZeV range from Space. Homage to the Discovery of Cosmic Rays. Nova Science Publishers, New York, ISBN: 978-162618-998-0, Inc, Pg 201-212 (2013)

[2] Rodríguez Frías, M.D. et al. for the JEM-EUSO Collaboration. The Atmospheric Monitoring System of the JEM-EUSO Space Mission. Proc. International Symposium on Future Directions in UHECR Physics, The European Physical Journal, Vol 53, 10005-pg1-7, http://dx.doi.org/10. 1051/epjconf/20135310005, (2013)

The JEM-EUSO Collaboration (corresponding authors S. Toscano, J.A. Morales de los Rios, A. Neronov, M.D. Rodríguez Frías \& S. Wada). The Atmospheric Monitoring System of the JEMEUSO instrument. Experimental Astronomy 37, DOI: $10.1007 / \mathrm{s} 10686-014-9378-1$ (2014)

[3] Sáez-Cano, G., Shinozaki, K., del Peral, L., Bertaina, M. and Rodríguez Frías, M.D. for the JEM-EUSO Collaboration. Observation of extensive air showers in cloudy conditions by the JEM-EUSO Space
Mission. Advance in Space Research, 53, 1536-1543 (2014)

[4] Sáez-Cano, G., Morales de los Rios, J.A., del Peral, L., Neronov, A., Wada, S. and Rodríguez Frías, M.D. for the JEM-EUSO Collaboration. Thin and thick cloud top height retrieval algorithm with the Infrared Camera and LIDAR of JEM-EUSO. These Procc

[5] http://www.ncep.noaa.gov/; http://gmao.gsfc.nasa.gov/; http: //www . ecmwf .int/

[6] Rodríguez Frías, M.D. et al. for the JEM-EUSO Collaboration. Towards the Preliminary Design Review of the Infrared Camera of the JEM-EUSO Space Mission. Proc. of 33rd International Cosmic Ray Conference (ICRC), Rio de Janeiro, Brazil (2013), [arXiv : 1307.7071v1] [astro-ph.IM] The JEM-EUSO Collaboration (corresponding authors J.A. Morales de los Rios \& M.D. Rodríguez Frías). The Atmospheric Monitoring System of the JEM-EUSO instrument. Experimental Astronomy 37, DOI: 10.1007/s10686-014-9402-5 (2014)

[7] Von Ballmoos, P., et al. A balloon-borne prototype for demonstrating the concept of JEM-EUSO. Advance in Space Research 53, 1544-1560 (2014). Morales de los Rios, J.A., Joven, E., del Peral, L., Reyes, M., Licandro, J., and Rodríguez Frías, M.D. The Infrared Camera Prototype Characterization for the JEM-EUSO Space Mission. Nuclear Instruments and Methods NIMA, 749, 74-83, ISSN: 0168-9002 (2014)

[8] D. Bayat, "Large Hybrid High Precision MEMS Mirrors", Ph.D. thesis, 2012 (http:// infoscience.epfl.ch/record/167903/files/ EPFL_TH5152.pdf)

[9] S. Toscano, L. Valore, A. Neronov, F. Guarino (JEMEUSO Collaboration), "LIDAR treatment inside the ESAF. "Simulation Framework for the JEM-EUSO mission", Proc. of 33rd International Cosmic Ray Conference (ICRC), Rio de Janeiro, Brazil (2013), ID0530. Preprint: [arXiv: 1307.7071] 